\title{
Renormalization of the energy-momentum tensor on the lattice
}

\section{Pepe*}

INFN, Sezione di Milano-Bicocca

Edificio U2, Piazza della Scienza 3

20126 Milano, Italy.

E-mail: Michele.Pepe@mib.infn.it

\section{Leonardo Giusti}

Dipartimento di Fisica, Università di Milano-Bicocca

and INFN, sezione di Milano-Bicocca

Edificio U2, Piazza della Scienza 3

20126 Milano, Italy.

E-mail: Leonardo.Giusti@mib.infn.it

We present the calculation of the non-perturbative renormalization constants of the energymomentum tensor in the $S U$ (3) Yang-Mills theory. That computation is carried out in the framework of shifted boundary conditions, where a thermal quantum field theory is formulated in a moving reference frame. The non-perturbative renormalization factors are then used to measure the Equation of State of the $S U$ (3) Yang-Mills theory. Preliminary numerical results are presented and discussed.

The European Physical Society Conference on High Energy Physics 22-29 July 2015

Vienna, Austria

\footnotetext{
* Speaker.
} 


\section{Introduction}

The energy-momentum tensor is a fundamental field of a Quantum Field Theory since it contains the currents associated to Poincaré symmetry and scale invariance. Furthermore, the expectation values of its matrix elements are physical quantities since they are directly related to the thermodynamic features of a quantum theory at finite temperature [1]. When the regularization of a quantum theory preserves space-time symmetries - like, for instance, dimensional regularization the energy-momentum tensor does not renormalize since it is related to a conserved current. However, non-perturbative investigations starting from first principles are usually carried out by considering the formulation of the quantum theory on the lattice. This regularization explicitly breaks the Poincaré invariance that is recovered only in the continuum limit. Thus, the energy-momentum tensor on the lattice is no longer trivially a conserved current and it needs to be properly renormalized to guarantee that the associated currents generate translations and rotations in the continuum limit [2]. For the Yang-Mills theory, scale invariance is also broken by the regularization; however, that symmetry is anomalous and it is not restored in the continuum limit, generating a dynamical mass-gap.

The approach that has been used to define a properly renormalized energy-momentum tensor on the lattice is to impose the validity of some Ward Identities at fixed lattice spacing up to terms that vanish when the lattice spacing goes to 0 [2]. Based on that framework, the renormalization of the energy-momentum tensor has been computed in perturbation theory at 1 loop [3]. Although one can in principle construct a lattice definition of the energy-momentum tensor, the non-perturbative calculation of the renormalization factors can be not straightforward if one has to consider correlation functions that are difficult to measure by numerical simulations.

A few years ago - based on an old suggestion by Landau [1] - it has been proposed to consider a thermal quantum field theory in a moving reference frame $[4,5,6]$. In the path-integral language, this can be accomplished by considering a spatial shift $\xi$ when closing the boundary conditions along the temporal direction. The shift $\xi$ corresponds to the Wick rotation of the speed of the moving frame. Interestingly, in this new framework, the parity symmetry is explicitly broken by the shift. This allows to write down new Ward Identities involving the energy-momentum tensor and new equations to measure both thermodynamic quantities and the renormalization factors. In particular, an off-diagonal matrix element of the energy-momentum tensor may have a non vanishing expectation value. Numerical simulations with shifted boundary conditions have already provided new successful, simple methods to study the thermodynamics of the Yang-Mills theory [7].

This report is organized as follows. In section 2, the main equations with shifted boundary conditions are summarized both in the continuum and on the lattice. The next section presents the results of the non-perturbative calculation of the renormalization factors of the energy-momentum tensor and, in section 4, the renormalization of the energy-momentum tensor is used to compute the Equation of State of the $S U$ (3) Yang-Mills theory. Conclusions and outlook follow.

\section{Shifted boundary conditions and Ward Identities}

In this section we consider the $S U$ (3) Yang-Mills theory at finite temperature in the Euclidean space. The path-integral formulation in a moving reference frame can be defined by considering 
shifted boundary conditions [6]

$$
A_{\mu}\left(L_{0}, \vec{x}\right)=A_{\mu}\left(0, \vec{x}-L_{0} \vec{\xi}\right)
$$

along the temporal direction of length $L_{0}$ with shift $\vec{\xi} \in \mathbb{R}^{3}$. When $\vec{\xi} \neq 0$, the parity symmetry is broken and there are new interesting Ward Identities involving the energy-momentum tensor $T_{\mu \nu}[4,5,6]\left(x_{0} \neq 0\right)$ :

$$
L_{0}\left\langle T_{0 k}\right\rangle_{\vec{\xi}}=\frac{1}{V} \frac{\partial}{\partial \xi_{k}} \ln Z\left(L_{0}, \vec{\xi}\right), \quad L_{0}\left\langle\bar{T}_{0 k}\left(x_{0}\right) O(0)\right\rangle_{\vec{\xi}, c}=\frac{\partial}{\partial \xi_{k}}\langle O\rangle_{\vec{\xi}}
$$

where $Z\left(L_{0}, \vec{\xi}\right)$ is the partition function with shifted boundary conditions and $O$ is a generic gauge invariant operator. The subscript $c$ indicates a connected correlation function, $\langle\cdot\rangle_{\vec{\xi}}$ stands for the expectation value with shifted boundary conditions and $\bar{T}_{\mu \nu}=\int d^{3} x T_{\mu v}(x)$. The field $T_{\mu \nu}$ can be defined by

$$
T_{\mu v}(x)=\frac{1}{g_{0}^{2}}\left[F_{\mu \rho}^{a}(x) F_{v \rho}^{a}(x)-\frac{1}{4} \delta_{\mu \nu} F_{\rho \sigma}^{a}(x) F_{\rho \sigma}^{a}(x)\right]
$$

where $g_{0}$ is the bare coupling constant and the field strength is given in terms of the gauge field $A_{\mu}(x)$ by $F_{\mu v}(x)=\partial_{\mu} A_{v}(x)-\partial_{v} A_{\mu}(x)-i\left[A_{\mu}(x), A_{v}(x)\right]$. Other useful equations are the following $[6,8]$

$$
\left\langle T_{0 k}\right\rangle_{\vec{\xi}}=\frac{\xi_{k}}{1-\xi_{k}^{2}}\left\{\left\langle T_{00}\right\rangle_{\vec{\xi}}-\left\langle T_{k k}\right\rangle_{\vec{\xi}}\right\}, \quad \frac{\partial}{\partial \xi_{k}}\left\langle T_{\mu \mu}\right\rangle_{\vec{\xi}}=\frac{1}{\left(1+\xi^{2}\right)^{2}} \frac{\partial}{\partial \xi_{k}}\left[\frac{\left(1+\xi^{2}\right)^{3}}{\xi_{k}}\left\langle T_{0 k}\right\rangle_{\vec{\xi}}\right] .
$$

When we consider the lattice regularization, the 10 dimensional symmetric $S O(4)$ representation of the energy-momentum tensor becomes reducible and it splits into the sum of the singlet, the triplet, and the sextet representations of the hyper-cubic group. The field $T_{\mu \nu}$ can then be expressed as a combination of the following three operators

$$
T_{\mu \nu}^{[1]}=\left(1-\delta_{\mu \nu}\right) \frac{1}{g_{0}^{2}} F_{\mu \alpha}^{a} F_{\nu \alpha}^{a} ; \quad T_{\mu \nu}^{[2]}=\delta_{\mu \nu} \frac{1}{4 g_{0}^{2}} F_{\alpha \beta}^{a} F_{\alpha \beta}^{a} ; \quad T_{\mu \nu}^{[3]}=\delta_{\mu \nu} \frac{1}{g_{0}^{2}}\left\{F_{\mu \alpha}^{a} F_{\mu \alpha}^{a}-\frac{1}{4} F_{\alpha \beta}^{a} F_{\alpha \beta}^{a}\right\}
$$

and the identity. Since translation and rotation symmetries are broken by the lattice regularization, the sextet $T_{\mu \nu}^{[1]}$ and the triplet $T_{\mu \nu}^{[3]}$ operators pick up a multiplicative renormalization factor, while the singlet $T_{\mu \nu}^{[2]}$ mixes in addition with the identity. The renormalized energy-momentum tensor can finally be written as $T_{\mu \nu}^{\mathrm{R}}=Z_{T}\left\{T_{\mu \nu}^{[1]}+z_{T} T_{\mu \nu}^{[3]}+z_{S}\left[T_{\mu \nu}^{[2]}-\left\langle T_{\mu \nu}^{[2]}\right\rangle_{0}\right]\right\}$.

Because of the finite renormalization factors, we can write the lattice version of the first equation of (2.2) as follows

$$
Z_{T}\left(g_{0}^{2}\right)=-\frac{\Delta f}{\Delta \xi_{k}} \frac{1}{\left\langle T_{0 k}^{[1]}\right\rangle_{\vec{\xi}}}, \quad \text { with } \quad \frac{\Delta f}{\Delta \xi_{k}}=\frac{1}{2 a V} \ln \left[\frac{Z\left(L_{0}, \vec{\xi}-a \hat{k} / L_{0}\right)}{Z\left(L_{0}, \vec{\xi}+a \hat{k} / L_{0}\right)}\right]
$$

The two equations in (2.4) are given by

$$
z_{T}\left(g_{0}^{2}\right)=\frac{1-\xi_{k}^{2}}{\xi_{k}} \frac{\left\langle T_{0 k}^{[1]}\right\rangle_{\vec{\xi}}}{\left\langle T_{00}^{[3]}\right\rangle_{\vec{\xi}}-\left\langle T_{k k}^{[3]}\right\rangle_{\vec{\xi}}}
$$




$$
z_{S}=\frac{1}{\left(1+\xi^{2}\right)^{2}} \frac{\left[\frac{\left(1+\xi^{\prime 2}\right)^{3}}{\xi_{k}^{\prime}}\left\langle T_{0 k}^{[1]}\right\rangle_{\vec{\xi}^{\prime}}\right]_{\vec{\xi}^{\prime}=\vec{\xi}+a \hat{k} / L_{0}}-\left[\frac{\left(1+\xi^{\prime 2}\right)^{3}}{\xi_{k}^{\prime}}\left\langle T_{0 k}^{[1]}\right\rangle_{\vec{\xi}^{\prime}}\right]_{\vec{\xi}^{\prime}=\vec{\xi}-a \hat{k} / L_{0}}}{\left\langle T_{\mu \mu}^{[2]}\right\rangle_{\vec{\xi}+a \hat{k} / L_{0}}-\left\langle T_{\mu \mu}^{[2]}\right\rangle_{\vec{\xi}-a \hat{k} / L_{0}}} .
$$

Note that the equations above allow for a fully non-perturbative definition of $T_{\mu v}$; furthermore they represent a simple procedure to perform the numerical calculation of $Z_{T}, z_{T}$ and $z_{S}$ since only the expectation values of local fields need to be measured.

\section{Numerical results}

In this section we present the results of Monte Carlo simulations to calculate $Z_{T}\left(g_{0}^{2}\right)$ and $z_{T}\left(g_{0}^{2}\right)$ in the range $g_{0}^{2} \in(0,1)[8]$; work is in progress for the computation of $z_{s}\left(g_{0}^{2}\right)$. We define the SU(3) Yang-Mills theory on a space-time lattice of volume $L^{3} \times L_{0}$ and lattice spacing $a$. We impose periodic boundary conditions in the spatial directions and shifted boundary conditions in the temporal direction: $U_{\mu}\left(L_{0}, \vec{x}\right)=U_{\mu}\left(0, \vec{x}-L_{0} \vec{\xi}\right)$, where $U_{\mu}\left(x_{0}, \vec{x}\right)$ are the link variables. We consider the standard Wilson action $S[U]=-1 / g_{0}^{2} \sum_{x, \mu v} \operatorname{Re} \operatorname{Tr}\left[U_{\mu v}(x)\right]$, where the plaquette is given by $U_{\mu v}(x)=U_{\mu}(x) U_{v}(x+a \hat{\mu}) U_{\mu}^{\dagger}(x+a \hat{v}) U_{v}^{\dagger}(x)$. The gluon field strength tensor is defined as [2]

$$
F_{\mu \nu}^{a}(x)=-\frac{i}{4 a^{2}} \operatorname{Tr}\left\{\left[Q_{\mu \nu}(x)-Q_{\nu \mu}(x)\right] T^{a}\right\},
$$

where $Q_{\mu v}(x)=U_{\mu v}(x)+U_{v-\mu}(x)+U_{-\mu-v}(x)+U_{-v \mu}(x)$, and the minus sign stands for the negative orientation. The renormalization constants $Z_{T}, z_{T}$ and $z_{S}$ are finite and depend on $g_{0}^{2}$ only. Considering the above definition of the field strength tensor on the lattice, at 1 loop in perturbation theory their expressions are [2,3]

$$
Z_{T}\left(g_{0}^{2}\right)=1+0.27076 g_{0}^{2}, \quad z_{T}\left(g_{0}^{2}\right)=1-0.03008 g_{0}^{2}, \quad z_{S}\left(g_{0}^{2}\right)=\frac{b_{0}}{2} g_{0}^{2} .
$$

\subsection{Computation of $Z_{T}$}

The direct determination of $\Delta f / \Delta \xi_{k}$ in (2.6) is a numerically challenging problem since it requires the computation of the ratio of two partition functions with a poor overlap of the relevant phase space $[9,10,4]$. Moreover, the calculation becomes quickly demanding for large lattices because the numerical cost increases quadratically with the spatial volume. Since $\Delta f / \Delta \xi_{k}$ is a smooth function of $g_{0}^{2}$ at fixed values of $L_{0} / a$ and $L / a$ in the range of chosen values, its derivative with respect to $g_{0}^{2}$ can be written as

$$
\frac{d}{d g_{0}^{2}} \frac{\Delta f}{\Delta \xi_{k}}=\frac{1}{2 a L^{3} g_{0}^{2}}\left\{\langle S\rangle_{\vec{\xi}-a / L_{0} \hat{k}}-\langle S\rangle_{\vec{\xi}_{+a / L_{0} \hat{k}}}\right\}
$$

The difference in the r.h.s. has been computed for $\vec{\xi}=(1,0,0)$ and $L / a=48$ at $L_{0} / a=3,4$ and 5 for many values of $g_{0}^{2}$. At each value of $L_{0} / a$ the points are interpolated with a cubic spline, and the resulting curve is integrated over $g_{0}^{2}$. The free-case value is computed analytically and is added to the integral. Then $\left\langle T_{0 k}^{[1]}\right\rangle_{\vec{\xi}}$ has also been computed at many values of $g_{0}^{2}$ and the results have been 
interpolated with cubic splines. The final result for $Z_{T}$ is shown in the left panel of Fig. 1 together with the 1-loop perturbative result and an interpolating fit

$$
Z_{T}\left(g_{0}^{2}\right)=\frac{1-0.4457 g_{0}^{2}}{1-0.7165 g_{0}^{2}}-0.2543 g_{0}^{4}+0.4357 g_{0}^{6}-0.5221 g_{0}^{8}
$$


Figure 1: The renormalization factor $Z_{T}\left(g_{0}^{2}\right)$ (left panel) and $z_{T}\left(g_{0}^{2}\right)$ (right panel) as a function of the bare coupling $g_{0}^{2}$. The dashed lines represent the 1-loop perturbative results and the solid lines are interpolating fits of the numerical data.

\subsection{Determination of $z_{T}$}

The renormalization constant $z_{T}$ is calculated by imposing the tree-level improved version of Eq. (2.7) given by $\left\{z_{T}\left(g_{0}^{2}\right)-\right.$ free case $\}$, with $\frac{L \xi_{k}}{L_{0}\left(1+\xi_{k}^{2}\right)}=q \in \mathbb{Z}$. The expectation values of $\left\langle T_{0 k}^{[1]}\right\rangle_{\vec{\xi}}$ and of the difference $\left\langle T_{00}^{[3]}\right\rangle_{\vec{\xi}}-\left\langle T_{k k}^{[3]}\right\rangle_{\vec{\xi}}$ are measured straightforwardly in the same simulation.

We chose $\vec{\xi}=(1 / 2,0,0)$ and $q=8$ so that the ratio of the spatial linear size over the temporal one is fixed to be $L / L_{0}=20$. We simulated 5 values of $g_{0}^{2}$ in the range $0 \leq g_{0}^{2} \leq 1$ with temporal length $L_{0} / a=4,6,8$ and 12 . After performing a combined extrapolation to $a / L_{0}=0$, the final results are shown in the right panel of Fig. 1. The dashed line is the 1-loop perturbative result and the solid line is an interpolating fit

$$
z_{T}\left(g_{0}^{2}\right)=\frac{1-0.5090 g_{0}^{2}}{1-0.4789 g_{0}^{2}}
$$

\section{A physical application: the Equation of State}

In this section we use the results of the non-perturbative renormalization of the energy-momentum tensor to obtain the Equation of State from Monte Carlo simulations. The energy-momentum tensor is a physical field and its expectation values are related to the thermodynamics features of a quantum field theory. In fact an interesting Ward Identity that follows using shifted boundary conditions is the following [6]

$$
\frac{s}{T^{3}}=-\frac{L_{0}^{4}\left(1+\xi^{2}\right)^{3}}{\xi_{k}}\left\langle T_{0 k}\right\rangle_{\xi} Z_{T}
$$


where $s$ is the entropy density and $T=1 / L_{0} \sqrt{1+\xi^{2}}$ is the temperature. In [7] the temperature dependence of $s / T^{3}$ has been measured using the step-scaling function: in that approach one can avoid computing $Z_{T}$ but only fixed, constant steps in the temperature can be done. Once the renormalization factor $Z_{T}$ is known, the eq. (4.1) allows to measure $s / T^{3}$ independently at any temperature; furthermore the extrapolation to the continuum limit is carried out in a very simple way.

In Fig. 2 we compare our preliminary data with the results available in the literature. In the region 1-2.5 $T_{c}$ we find a discrepancy with [12] and our data are compatible with [11]. At larger temperatures up to $T \simeq 7 T_{c}$, our results agree with those presented in [12]. Work is in progress to clarify the above mentioned discrepancy and to reach temperatures about $250 T_{c}$.

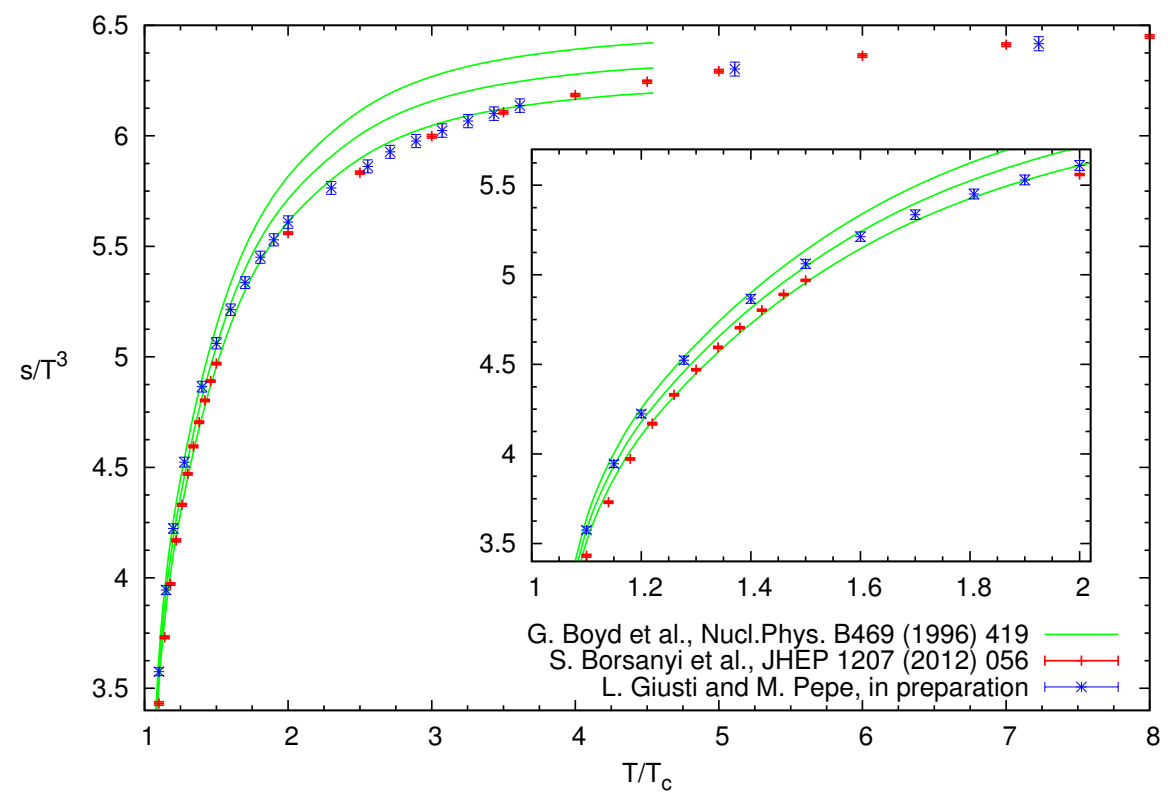

Figure 2: The temperature dependence of the dimensionless ratio $s / T^{3}$. The results obtained using eq. (4.1) are compared with data available in the literature.

\section{Conclusions and outlook}

We have presented results for the non-perturbative renormalization of the energy-momentum tensor on the lattice. In particular, the renormalization factors of the traceless diagonal and offdiagonal components of $T_{\mu \nu}$ have been calculated. An equation to compute the renormalization factor of the trace is also presented and work is in progress to perform that measurement. The physical relevance of the non-perturbative renormalization of the energy-momentum tensor is discussed presenting the results of Monte Carlo simulations to compute the Equation of State of the $S U$ (3) Yang-Mills theory. The framework of shifted boundary conditions turns out to be very effective to investigate the Yang-Mills theory at finite temperature. Work is in progress to include also dynamical fermions.

Acknowledgements. M.P. wishes to thank K. Szabo for the invitation to the Conference. 


\section{References}

[1] L. Landau and E. Lifshitz, Course of Theoretical Physics VI: Fluid Mechanics, Butterworth-Heinemann (1987).

[2] S. Caracciolo, G. Curci, P. Menotti and A. Pelissetto, Annals Phys. 197, 119 (1990).

[3] S. Caracciolo, P. Menotti and A. Pelissetto, Nucl. Phys. B 375, 195 (1992).

[4] L. Giusti and H. B. Meyer, Phys. Rev. Lett. 106, 131601 (2011).

[5] L. Giusti and H. B. Meyer, JHEP 1111, 087 (2011).

[6] L. Giusti and H. B. Meyer, JHEP 1301, 140 (2013).

[7] L. Giusti and M. Pepe, Phys. Rev. Lett. 113, 031601 (2014).

[8] L. Giusti and M. Pepe, Phys. Rev. D 91, 114504 (2015).

[9] P. de Forcrand, M. D’Elia and M. Pepe, Phys. Rev. Lett. 86, 1438 (2001).

[10] M. Della Morte and L. Giusti, Comput. Phys. Commun. 180, 813 (2009).

[11] G. Boyd, J. Engels, F. Karsch, E. Laermann, C. Legeland, M. Lutgemeier and B. Petersson, Nucl. Phys. B 469, 419 (1996).

[12] S. Borsanyi, G. Endrodi, Z. Fodor, S. D. Katz and K. K. Szabo, JHEP 1207, 056 (2012). 